\title{
Spatial Temporal based Classification for Antebrachium and Carpus Movement of EEG Data using Emotive Head Set
}

\author{
Muhammad Ahsan Gull ${ }^{1}$, Javaid Iqbal ${ }^{2}$ and Mohsin I. Tiwana \\ National University of Sciences \& Technology, Islamabad, Pakistan. \\ 1ahsangul74@mts.ceme.edu.pk; 2j.iqbal@ceme.nust.edu.pk
}

\begin{abstract}
Electroencephalographic (EEG) signals from test subjects are used for $\mathrm{BCl}$ analysis and classification of the four upper limb movements. Brain computer interface (BCl) system provides better neural control to the user, over two antebrachium and two carpus movement. This paper uses Epoc Emotiv head set with fourteen electrodes to acquire signal from the motor area of scalp. A particular protocol for data acquisition is followed. Our method is based on the time domain feature extraction techniques. Activity feature is applied on the signals. In order to discriminate between four upper limb movements within the data set, the paper uses Quadratic support vector machine, RBF SVM and Multilayer perceptron. The best classification accuracy of two antebrachium and two carpus movement is achieved by Quadratic SVM and RBF SVM when Gaussian window is used to calculate the activity feature vector. The number of correctly classified instants in term of percentage is $76.92 \%$ and $75.96 \%$ for quadratic SVM and RBF SVM. Current classifiers show promising results, where as to make conclusion more generalized and to enhance the classification accuracy, further experiments need to be carried out.
\end{abstract}

Keywords: Classification, Multilayer perceptron, Support Vector Machine, Hjorth Parameters.

\section{Introduction}

Brain Computer Interface provides us with a communication facility to actuate device using electroencephalographic signals. Currently, $\mathrm{BCl}$ is used to deliver commands to the processor, which transforms commands into certain specified actions. It's an emerging, fast-growing technology and is being used now a day for numerous controlling purposes. In this paper, experimental setup uses emotive headset for the collection of EEG data [8]. The main advantage of using emotive headset is that it is not only a noninvasive $\mathrm{BCl}$ technique to collect EEG data, but also provides better portability with fourteen active electrodes. A clinical survey showed that most of the causalities or strokes results in the disability of the people. Such a type of disability can be addressed either by restoring motor function of

Data acquisition and offline analysis are supported by Department of Mechatronics Engineering (College of Electrical \& Mechanical Engineering) National University of Sciences \& Technology Pakistan. The research is carried out under the supervision of Javaid Iqbal and Mohsin I. Tiwana. This publication only reflects the author s' views.

DOI: $10.14738 /$ jbemi.16.813

Publication Date: $5^{\text {st }}$ January 2015

URL: http://dx.doi.org/10.14738/jbemi.16.813 
patients, or by providing them a prosthetic device [1]. Currently, evoked potential in combining with the robotic feedback is a sound technique to address the motor disability of the people [1]. Most important and recent research applications of $\mathrm{BCl}$ are controlling smart home appliances [2], wheel chair control, mobile robot control, controlling a prosthetic robotic arm, etc. [3] - [7].

This paper will classify the offline EEG data for two carpus and two antebrachium movements. Extensive literature review is carried out to classify upper limb movement from EEG data using different protocols and experimental setups. Table 1 shows brief survey of classification and feature extraction techniques which have been used to classify the different upper limb movements.

Table 1: Literature Review

\begin{tabular}{|c|c|c|c|}
\hline References & Classifiers & Feature & $\%$ age Accuracy \\
\hline $\begin{array}{l}\text { G. N. Garcia } \\
\text { T. Ebrahimi } \\
\text { J.-M. Vesin [9] }\end{array}$ & $\begin{array}{c}\text { Gaussian Support } \\
\text { vector machine } \\
\text { Linear Discernment Analysis }\end{array}$ & $\begin{array}{c}\text { Correlative time frequency } \\
\text { based feature }\end{array}$ & $\begin{array}{l}86.00 \% \\
61.00 \%\end{array}$ \\
\hline $\begin{array}{c}\text { S. Solhjoo } \\
\text { M. H. Moradi [10] }\end{array}$ & $\begin{array}{l}\text { Gaussian Classifier } \\
\text { Linear Discriminants } \\
\text { Analysis } \\
\text { Bayes Quadratic } \\
\text { Mahalanobis distance }\end{array}$ & $\begin{array}{l}\text { Power Spectral } \\
\text { Density }\end{array}$ & $\begin{array}{l}65.4 \% \\
65.6 \% \\
63.4 \% \\
63.3 \%\end{array}$ \\
\hline $\begin{array}{c}\text { H. Lee } \\
\text { S. Choi, [11] }\end{array}$ & $\begin{array}{c}\text { Support vector } \\
\text { Machine + HMM } \\
\text { Hidden Markov Model }\end{array}$ & $\begin{array}{c}\text { Principal Component } \\
\text { Analysis }\end{array}$ & $\begin{array}{l}78.15 \% \\
75.70 \%\end{array}$ \\
\hline $\begin{array}{c}\text { Weibo Yi } \\
\text { Shuang Qiu } \\
\text { Hongzhi Qi } \\
\text { Lixin Zhang } \\
\text { Baikun Wan } \\
\text { Dong Ming, [12] }\end{array}$ & $\begin{array}{l}\text { Support vector } \\
\text { machine }\end{array}$ & $\begin{array}{c}\text { Multi-CSP } \\
\text { Multi-GECSP } \\
\text { Multi-sTRCSP }\end{array}$ & $\begin{array}{l}70.07 \% \\
68.73 \% \\
70.43 \%\end{array}$ \\
\hline $\begin{array}{c}\text { Ricardo } \\
\text { C.Caracillo } \\
\text { Maria Claudia } \\
\text { F. Castro, [13] }\end{array}$ & $\begin{array}{l}\text { Linear Discriminant } \\
\text { Analysis }\end{array}$ & $\begin{array}{l}\text { Power Spectral } \\
\text { Density }\end{array}$ & $\begin{array}{c}83.69 \% \\
\text { (Hand v/s arm) } \\
67.95 \% \\
\text { (Right v/s Left } \\
\text { limb) }\end{array}$ \\
\hline $\begin{array}{l}\text { J. Sleight } \\
\text { P. Pillai } \\
\text { S. Mohan, [14] }\end{array}$ & Support Vector Machine & $\begin{array}{l}\text { Power Spectral Feature in } \\
\text { Alpha beta \& theta band }\end{array}$ & $64.00 \%$ \\
\hline
\end{tabular}

This paper focuses on the noninvasive $\mathrm{BCI}$ technique, in which the $\mathrm{EEG}$ data is collected from a test subject using Emotive head set equipped with fourteen active electrodes. Data is recorded for each particular action for eight seconds. Once the data is recorded, fourth order Butterworth filter is applied for removal of unwanted artifacts and noise from the data set. For further rejection of undesired artifacts and to make the data unique to achieve the better classification of carpus-up, carpus-down, antebrachium-up, and antebrachium-down movements, it is a better practice to use windowing on data set for processing. Further, this paper uses supervised learning classification techniques (the quadratic 
Muhammad Ahsan Gull, Javaid Iqbal and Mohsin I. Tiwana; Spatial Temporal based Classification for Antebrachium and Carpus Movement of EEG Data using Emotive Head Set. Journal of Biomedical Engineering and Medical Imaging, Volume 1, No. 6, Dec (2014) , pp 67-75

SVM, radial based SVM and Multi-layer perceptron) to classify four upper limb movements when activity (hjorth feature) is used as a feature extraction technique. Figure 1 shows schematic diagram for the whole process.

\section{Experimental Protocol and Data Acquisition}

An experimental protocol has been design to acquire the data for offline analysis. Two right handed subjects (age 25 and 26) participates in the data acquisition experimentation. Both subjects are neurologically stable and physically healthy. The experimental protocol consists of four right handed tasks (antebrachium-upward movement, antebrachium-downward movement, carpus-upward movement and carpus-downward movement). The subjects are instructed to avoid any facial expression or eye flickering in order to avoid unwanted artifacts while performing the tasks. Thirty trials with 1051 samples in 8.29 seconds are recorded from each test subject. The data from each test subject is recorded in a single trial whereas initial data set for a particular move is separated by markers. Mode of protocol used was P300.

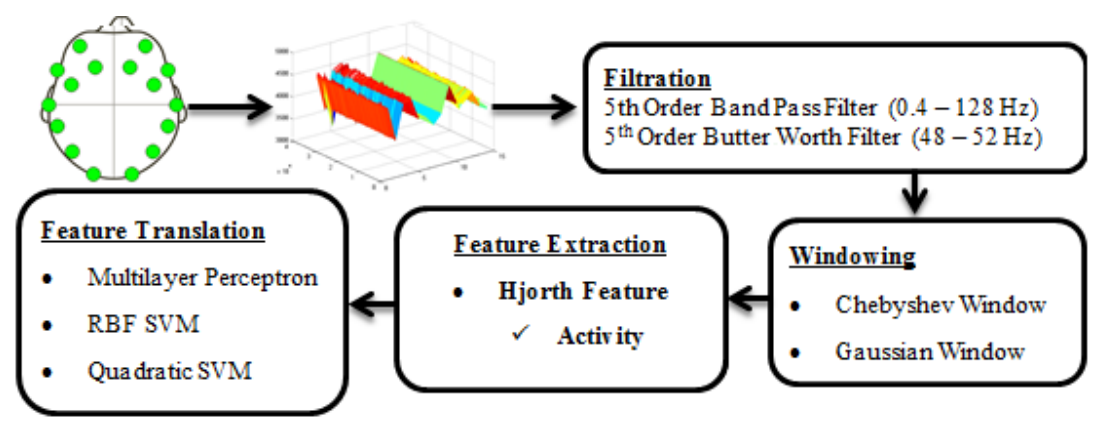

Figure 1: Schematic Diagram of Brain Computer Interface System

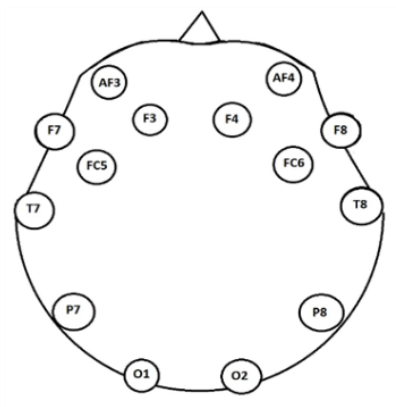

Figure 2: 14 Electrodes locations are shown

Emotiv Epoc neurohead set was used to acquire EEG data from the test subjects. These days, the research society uses EEG technology to classify and accomplish different tasks due to its prominent advantages over other technology as discussed in the Table 2. 
Journal of Biomedical Engineering and Medical Imaging, Volume 1, Issue 6, December, 2014

Table 2: Brain sensing technologies and their primary disadvantages in $\mathrm{BCl}$ research

\begin{tabular}{|c|c|}
\hline Technology & Primary Disadvantage \\
\hline Electrocorticogram (ECoG) & Highly invasive, surgery \\
\hline Magneto-encephalography (MEG) & Extremely expensive \\
\hline Computed Tomography (CT) & Only anatomical data \\
\hline $\begin{array}{c}\text { Single Photon Emission Computerized Tomography } \\
\text { (SPECT) }\end{array}$ & Radiation exposure \\
\hline Positron Emission Tomography (PET) & Radiation exposure \\
\hline Magnetic Resonance Imaging (MRI) & Only anatomical data \\
\hline $\begin{array}{c}\text { Functional Magnetic Resonance Imaging (fMRI) } \\
\text { Event-Related Optical Signal / Functional Near-Infrared } \\
\text { (EROS/fNIR) }\end{array}$ & $\begin{array}{c}\text { Still in infancy, currently } \\
\text { expensive }\end{array}$ \\
\hline
\end{tabular}

By using Emotiv headset, data is recorded as per designed protocol. This EEG headset is equipped with 14 active electrodes use to acquire evoked potential from scalp. The sequential sampling rate of data is $2048 \mathrm{~Hz}$ and then the device down sample the data at $128 \mathrm{~Hz}$. Finally, the incoming data is filtered by fifth order sinc notch filter. Table 3 discusses the necessary specifications of Emotiv headset and figure 2 shows the location of 14 sensors over scalp.

Table 3: EMOTIV EPOC Neuro headset Specification [8]

\begin{tabular}{|c|c|}
\hline No. of Channels & 14 (plus CMS/DRL references, P3/P4 locations) \\
\hline Channels Name & AF3, AF4, F3, F4, F7, F8, FC5, FC6, P7, P8, T7, T8, 01, 02. \\
\hline Sampling Rate & $\sim 128 \mathrm{~Hz}$ \\
\hline Sampling method & Sequential sampling. Single ADC \\
\hline Resolution & 14 bits $1 \mathrm{LSB}=0.51 \mu \mathrm{V}(16$ bit ADC, 2 bits instrumental noise floor discarded) \\
\hline Filtering & Built in digital 5th order Sinc filter \\
\hline Dynamic range & $8400 \mu \mathrm{V}(\mathrm{pp})$ \\
\hline Coupling mode & AC coupled \\
\hline Connectivity & Proprietary wireless, 2.4GHz band \\
\hline Power & LiPoly batteries \\
\hline Battery life & 12 hours \\
\hline
\end{tabular}

\section{Filtration and Windowing}

Once the data is acquired, it is band pass filtered $(0.4-128 \mathrm{~Hz})$ with $500 \mathrm{~Hz}$ sampling frequency and fifth order butter worth filter $(48 \mathrm{~Hz}-52 \mathrm{~Hz})$ is used to clip the baseline noise. In $\mathrm{BCl}$, the aim of filtration is to remove the unwanted artifacts recorded during data acquisition [13]. After filtration, a better practice to extract the feature is to make a window on the data set. The size of window should be carefully selected 
Muhammad Ahsan Gull, Javaid Iqbal and Mohsin I. Tiwana; Spatial Temporal based Classification for Antebrachium and Carpus Movement of EEG Data using Emotive Head Set. Journal of Biomedical Engineering and Medical Imaging, Volume 1, No. 6, Dec (2014) , pp 67-75

because larger window can accommodate outlier while calculating the feature and much smaller window can lose confidence. In this paper, the window applied on the data set is of 52X1 with $0.1 \mathrm{sec}$ step size [13]. Two different types of windows (Gaussian window and Chebyshev window) were applied on the data set for analysis as shown in the figure 3.
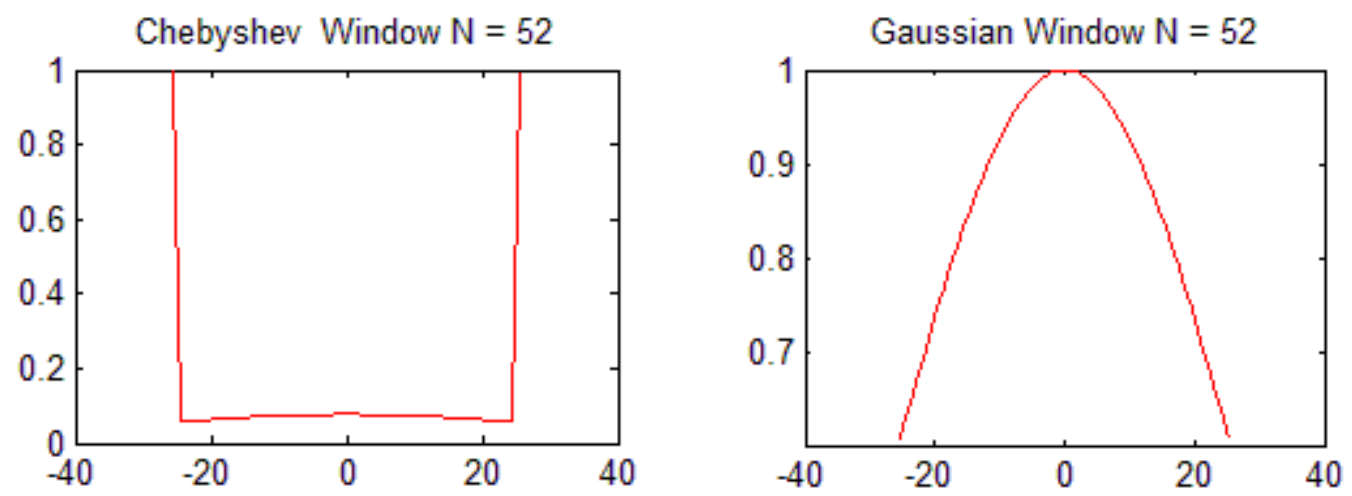

Figure 3: Gaussian window of $\mathbf{N}=\mathbf{5 2}$ samples and alpha $=1$. Here alpha is inversely proportional to

Standard deviation. And Chebyshev window of 52 samples with side lobe magnitude $r=9$

\section{Feature Translation}

A Time domain feature extraction technique, Activity (also known as one of the hjorth features) is used for the reduction of artifacts. Different artifacts removal techniques are used by various authors as mentioned in the Table no. 1. In BCl, the aim of feature extraction is to remove the unwanted artifacts recorded during data acquisition [10] like eye blinking, other unwanted muscular activity and etc.

$$
y\left(x_{i}\right)=\sqrt{\frac{\operatorname{var}\left(x_{i}^{\prime}\right)}{\operatorname{var}\left(x_{i}\right)}}
$$

$x_{i}$ represents the signal, $x_{i}{ }^{\prime}$ is the rate of change of signals and $y\left(x_{i}\right)$ is the measure of signal's mean frequency. A hjorth approach, activity is also used in this paper to find the attributes. Mathematically it can be represented as.

$$
\operatorname{Activity}(x)=\frac{\sum_{n=1}^{n}\left(x_{n}-\bar{x}\right)^{2}}{N}
$$

$X_{n}$ is nth number of sample in the data set and is the mean and $N$ be the total number of samples. Activity calculates that how much signal is deviated from its mean value. The topographical distribution of feature vector is shown in the figure4. There are different frequency domain methods for calculating the feature vector like PSD, BP, wavelet, Fourier, AR and etc. but they have complex parameters and causes to enhance over fitting difficulties while classifying dat.

\section{Classification Techniques}

Once feature vector have calculated now classify the data depending upon the feature. This paper used one against one technique for RBF support vector machine and quadratic SVM to classify the four upper limb movements by constructing a nonlinear hyper plane in feature space. Mathematically support vector machine is represented by following equation.

$$
c=\sum_{i} a_{i} k\left(s_{i}, x\right)+b
$$


$s_{i}$ is support vector, $a_{i}$ is weight and $\boldsymbol{b}$ is the bias that is used to classify the feature vector $\boldsymbol{x}$. Here $\boldsymbol{k}$ is the kernel function. Two types of kernel functions are used in this paper radial based kernel and quadratic kernel to separate the data. Equation 5 and 6 shows the mathematical representation of radial based function and quadratic function respectively.

$$
\begin{gathered}
k\left(s_{i} \cdot x\right)=e^{-\left\|s_{i}-x\right\|^{2}} \\
k\left(s_{i} \cdot x\right)=\left(s_{i} \cdot x+r\right)^{2} \quad \text { Where } r \geq 0
\end{gathered}
$$

$\boldsymbol{r}$ is the quadratic function parameter needed to be selected carefully for better classification. The classification accuracy of upper limb movement using support vector machine is shown in [12] and [14]. While [11] uses cascading of SVM with hidden markov model to classify the forearm movement. Another paradigm used to classify the two antebrachium and two carpus movement used in this paper is multi-layer perceptron. Its formulation is as follows.

$$
y=f(a)\left(\sum_{i=1}^{k}\left(w_{i} x_{i}+d\right)\right)
$$

$\boldsymbol{f}(\boldsymbol{a})$ is the activation function, $\boldsymbol{d}$ is the bias, $\boldsymbol{w}$ is the weight and $\boldsymbol{x}$ is the feature vector. Using $\mathrm{BCl}$ competition IV, A dataset for three motor imageries (left hand, right hand and right foot), multilayer perceptron shows $85.71 \%$ classification accuracy with 0.1 learning rate [16].

\section{Results and Discussion}

The overall classification rates of above mentioned classifier with activity as a feature are shown in the table 4. Based on the literature survey, analysis, and results obtained, it can be concluded that Quadratic support vector machine and radial based function support vector machine give the best classification accuracy of $76.92 \%$ and $75.96 \%$ when Gaussian window of 0.1 millisecond is used to find the activity feature vector. The analysis of Quadratic support vector machine and RBF SVM for the classification of four upper limb movement using activity (hjorth feature) presented in this paper is novel. Whereas better classification of antebrachium downward movement, antebrachium upward movement, Carpus downward movement and carpus upward movement is $92.31 \%, 80.70 \%, 63.00 \%$ and $80.70 \%$, respectively, using activity with quadratic SVM. RBF SVM and quadratic SVM with activity feature shows much better results (shown in the table4) as compared to the SVM with principal component analysis, power spectral density, Multi-CSP Multi GECSP and Multi-sTRCSP used as the feature extraction techniques. whereas literature survey reports the classification accuracy of upper limb movement using SVM with principal component analysis [11], power spectral density [14], Multi-CSP [12], Multi GECSP [12] and Multi-sTRCSP [12] is 78.15\%, 64.00\%, 70.07\%, 68.73\% and 70.43\% respectively. Table 4 expresses the classification accuracy of three classifiers. Best classification accuracy of multilayer perceptron is $72.06 \%$ when Gaussian window is used for calculating activity feature vector. By applying Chebyshev window it is found that MLP shows $70.43 \%$ correctly classified instants. Figure 4 shows the topographical distribution of four upper limb movements. In figure 4 the red area shows convergence of the data over the left hemisphere of frontal lobe of motor cortex, the maps are made based on the activity (hjorth value) values of each electrode. 
Muhammad Ahsan Gull, Javaid Iqbal and Mohsin I. Tiwana; Spatial Temporal based Classification for Antebrachium and Carpus Movement of EEG Data using Emotive Head Set. Journal of Biomedical Engineering and Medical Imaging, Volume 1, No. 6, Dec (2014) , pp 67-75

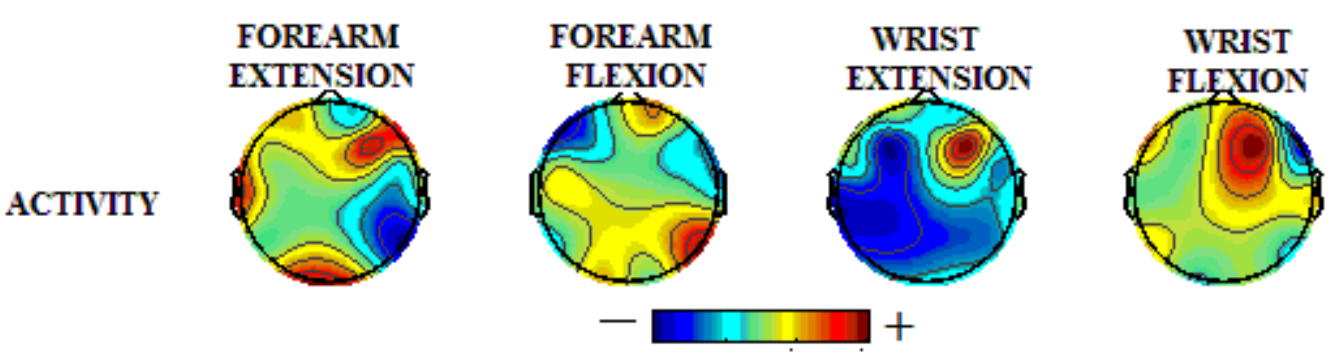

Figure 4: Topoplots of feature vector (Activity) for Brachium Down movement, Brachium Up movement, Carpus Down movement and Carpus up movement

Table 5 shows stratified cross validation results and weighted average accuracy of multilayer perceptron. These results shows some deviation and this may be due to the reason that if the size of classes differs it causes misclassification and the number of false positives increases. This research has great importance, due to the reason that one can understand that how specific neural activity is differ from the other motor area. In order to enhance true positive and make the solution more generic it is necessary to increase number of trials by increasing number of test subjects.

Table 4: Percentage Accuracy of Classifier with Activity feature for four upper limb movement.

\begin{tabular}{|c|c|c|}
\hline $\begin{array}{c}\text { Classification } \\
\text { Algorithm }\end{array}$ & $\begin{array}{c}\text { Gaussian } \\
\text { Window }\end{array}$ & $\begin{array}{c}\text { Chebyshev } \\
\text { Window }\end{array}$ \\
\hline Quadratic SVM & $76.92 \%$ & $73.08 \%$ \\
\hline RBF SVM & $75.96 \%$ & $72.52 \%$ \\
\hline MLP & $72.60 \%$ & $70.43 \%$ \\
\hline
\end{tabular}

Table 5: Stratified cross validation results and weighted average accuracy by class.

\begin{tabular}{|c|c|c|c|c|c|c|c|}
\hline $\begin{array}{c}\text { Multilayer } \\
\text { Perceptron } \\
\text { (MLP) }\end{array}$ & $\begin{array}{c}\text { Kappa } \\
\text { Statistic }\end{array}$ & $\begin{array}{c}\text { Mean } \\
\text { Absolute } \\
\text { Error }\end{array}$ & $\begin{array}{c}\text { Relative } \\
\text { Absolute } \\
\text { Error }\end{array}$ & $\begin{array}{c}\text { Coverage } \\
\text { of Class } \\
\mathbf{( 0 . 9 5} \\
\text { level) }\end{array}$ & $\begin{array}{c}\text { Mean rel. } \\
\text { Region } \\
\text { Size } \\
\mathbf{( 0 . 9 5} \\
\text { level) }\end{array}$ & $\begin{array}{c}\text { False } \\
\text { Positive } \\
\text { (Weighted } \\
\text { Avg.) }\end{array}$ & $\begin{array}{c}\text { True } \\
\text { Positive } \\
\text { (Weighted } \\
\text { Avg.) }\end{array}$ \\
\hline Gaussian Window & 0.6346 & 0.168 & $44.80 \%$ & $95.67 \%$ & $50.18 \%$ & 0.726 & 0.091 \\
\hline Chebyshev Window & 0.6058 & 0.1724 & $45.97 \%$ & $93.02 \%$ & $47.59 \%$ & 0.704 & 0.099 \\
\hline
\end{tabular}

7 Conclusion

The current $\mathrm{BCl}$ research is focused on the optimization of classifier algorithms and better neural plasticity. In this study, the performance of Quadratic support vector machine, RBF support vector machine and Multilayer Perceptron using activity feature for the optimized classification of antebrachium upward-movement, antebrachium-downward movement, carpus-downward movement and carpus-upward movement has been investigated and analyzed. In this analysis, the best results achieved by Quadratic SVM and RBF SVM classifiers are $76.92 \%$ and $75.96 \%$ when activity is used as feature. Future work is aimed at enhancing the performance of algorithm to attain better classification results and to make the solution more generic by enhancing the number of data samples and number of test subjects. 


\section{REFERENCES}

[1]. Kai Keng Ang; Cuntai Guan; Kok Soon Phua; Chuanchu Wang, Irvin; The Chang Wu Chen; Effie Chew:" Transcranial direct current stimulation and EEG-based motor imagery $\mathrm{BCl}$ for upper limb stroke rehabilitation" 34th Annual International Conference of the IEEE EMBS San Diego, California USA, 28 August - 1 September, 2012.

[2]. Wei Tuck Lee; Humaira Nisar; Aamir S. Malik; Kim Ho Yeap, "A Brain Computer Interface for Smart Home Control” 2013 IEEE 17th International Symposium on Consumer Electronics (ISCE)

[3]. L. R. Hochberg; M. D. Serruya; G. M. Friehs; J. A. Mukan; M. Saleh,A. H. Caplan; A. Branner; D. Chen; R. D. Penn; and J. P. Donoghue. "Neuronal ensemble control of prosthetic devices by a human with tetraplegia." Nature, vol. 442, no. 13, pp. 164-171, Jul. 2006

[4]. S. M. T. Müller; W. C. Celeste; T. F. Bastos-Filho; and M. Sarcinelli- Filho. "Brain-computer Interface Based on Visual Computer Interface Based on Visual Evoked Potentials to Command Autonomous Robotic Wheelchair." Journal of Medical and Biological Engineering, vol. 30, no. 6, pp. 407-416, 2010.

[5]. Birbaumer N; Ghanayim N; Hinterberger T; Iversen I, Kotchoubey B, Ku "bler A, Perelmouter J, Taub E, Flor H. “A spelling device for the paralyzed". Nature 398:297-298. doi:10.1038/18581, 1999.

[6]. M. Asghari Oskoei and H. Hu. "Support vector machine-based classification scheme for myoelectric control applied to upper limb" IEEE Transactions on Biomedical Engineering, vol. 55, no. 8, pp. 1956-1965, 2008.

[7]. Wolpaw JR; Birbaumer N; McFarland DJ; Pfurtscheller G; Vaughan TM. "Brain-computer interfaces for communication and control." Clin Neurophysiol 113:767-791. doi: 10.1016/S1388-2457(02)00057-3, 2002.

[8]. Emotiv.com (2013). EPOC Features. [online] Retrieved from: http://www.emotiv.com/epoc/ [Accessed: 5 Mar 2013].

[9]. G. N. Garcia; T. Ebrahimi and J.-M. Vesin. "Support vector EEG classication in the Fourier and time-frequency correlation domains." In Conference Proceedings of the First International IEEE EMBS Conference on Neural Engineering, 2003.

[10]. S. Solhjoo and M. H. Moradi. "Mental task recognition: A comparison between some of classication methods." In BIOSIGNAL 2004 International EURASIP Conference, 2004

[11]. H. Lee and S. Choi. "Pca+hmm+svm for eeg pattern classification." In Proceedings of the Seventh International Symposium on Signal Processing and Its Applications, 2003. 
Muhammad Ahsan Gull, Javaid Iqbal and Mohsin I. Tiwana; Spatial Temporal based Classification for Antebrachium and Carpus Movement of EEG Data using Emotive Head Set. Journal of Biomedical Engineering and Medical Imaging, Volume 1, No. 6, Dec (2014) , pp 67-75

[12]. Yi et al.: "EEG feature comparison and classification of simple and compound limb motor imagery." Journal of NeuroEngineering and Rehabilitation 2013 10:106.

[13]. Ricardo C.Caracillo and Maria Claudia F. Castro. "Classification of Executed Upper Limb Movements by Means of EEG." In Biosignals and Biorobotics Conference (BRC), 2013 ISSNIP.

[14]. J. Sleight, P. Pillai, and S. Mohan, "Classification of Executed and Imagined Motor Movement EEG Signals," Ann Arbor, p. 10, 2009.

[15]. Johnny Lee and Desney Tan. "Using a low-cost electroencephalograph for task classification in hci research." UIST'06: Proceedings of the 19th annual ACM symposium on User interface software and technology, Oct 2006.

[16]. G.V. Sridhar and Dr. P. Mallikarjuna Rao "A Neural Network Approach for EEG classification in BCl." International Journal of Computer Science and Telecommunications [Volume 3, Issue 10, October 2012] 